\title{
Gingival inflammation and platelet count in patients with leukemia: preliminary results
}

Patrícia Daniela Melchiors Angst ${ }^{(a)}$ Danilo Antônio Milbradt Dutra(b) Carlos Heitor Cunha Moreira ${ }^{(b)}$ Karla Zanini Kantorski(b)

(a) Department of Periodontology, Dental School, Universidade Federal do Rio Grande do Sul, Porto Alegre, RS, Brazil.

(b) Division of Periodontology, Department of Stomatology, Dental School, Universidade Federal de Santa Maria, Santa Maria, RS, Brazil.
Declaration of Interests: The authors certify that they have no commercial or associative interest that represents a conflict of interest in connection with the manuscript.

Corresponding author:

Patrícia Daniela Melchiors Angst

Email: pati_dani@hotmail.com

Received for publication on Aug 10, 2011 Accepted for publication on Oct 10, 2011

\begin{abstract}
Leukemia has been associated with oral manifestations. However, the available literature on this topic consists of mostly reports of cases, without data about the periodontal parameters that may be under the influence of hematologic factors. The aim of this cross-sectional study was to assess the correlation between the Gingival Index and Bleeding on Probing with the platelet count in patients with leukemia. Patients with diagnosis of any kind of leukemia, at any stage of treatment, having a minimum age of 14 years, treated at the Department of Hematology-Oncology of the University Hospital of Santa Maria, Brazil, between December 2009 and March 2010, were assessed. Excluded patients were: edentulous, with orthodontic appliances, with psychomotor disturbances, requiring antibiotic prophylaxis for the examinations, or those using medications associated with gingival swelling. Two trained and calibrated examiners evaluated the Plaque Index, Gingival Index (GI), Probing depth, Bleeding on Probing (BOP), and Clinical Attachment Loss. Hematologic data were collected from a blood test performed on the same day as the periodontal examination. Thirty-seven patients (26 males), aged between 15 and 80 years (mean age $41.7 \pm 18.31$ ) were evaluated. Correlation between platelet count and BOP ( $p>0.05)$, or between platelet count and GI ( $\mathrm{p}>0.05)$, were both weak (Pearson's correlation coefficient $r=0.171$ and $r=-0.003$, respectively) and not statistically significant. It can be concluded from the preliminary results that the low platelet count was not correlated with the higher prevalence of gingival and periodontal bleeding in patients with leukemia.
\end{abstract}

Descriptors: Leukemia; Platelet Counts; Periodontal Index.

\section{Introduction}

Leukemia is a malignancy of hematologic origin, occurring from either disorganized proliferation or increased life span of white cells (now called blasts). Leukemia can be classified basically as lymphoid or myeloid, according to the cell lineage, and as acute or chronic, according to the evolution of the disease. ${ }^{1-3}$ Due to the growing accumulation of blasts initially in the bone marrow, other hematopoietic cells (erythrocytes, macrophages/monocytes, eosinophils, basophils and lymphocytes) are suppressed. As the disease progresses, the excess blasts may spread to the bloodstream and infiltrate organs and tissues. ${ }^{2-5}$

Worldwide, estimates of incidence are 257,000 new cases per year 
(about $56 \%$ of which are men). However, the mean cumulative 5 -year survival rate is only $45 \%$ of cases in developed countries and only one third of this rate in developing countries. ${ }^{6}$

Thrombocytopenia, a common sign found in leukemic patients and due to either a reduced production of platelets or suppression of the normal red cells, frequently leads to episodes of hemorrhages. In this sense, thrombocytopenia is considered an important secondary effect of the disease and a potential risk factor. ${ }^{3-5}$

Oral manifestations (gingival swelling ${ }^{7-12}$ and gingival and oral bleeding ${ }^{13-18}$ ) have been associated with leukemia. Gingival swelling is the most common oral sign in untreated patients. ${ }^{7}$ On the other hand Hou et al., ${ }^{18}$ found that gingival bleeding is the more common initial oral sign in both acute and chronic leukemia.

However, these findings are based mostly on either case reports ${ }^{1,4,5,8-12,16,17,19}$ or retrospective data. ${ }^{7,13,14,18}$ These data are often collected by nondental health professionals, who do not provide adequate information about the periodontal status of this group of patients. A limited number of studies $^{20-22}$ have reported the prevalence of periodontal parameters in these individuals, and then only in patients with the acute form of the disease. Little is known about the periodontal status of these patients, so there is no strong scientific evidence to substantiate that the systemic status of patients with leukemia can influence their periodontal condition.

A cross-sectional design with high response rates can provide data that allow more valid and precise inferences about the parameters assessed and, accordingly, may allow for verification of possible associations. ${ }^{23}$

Therefore, this study aimed to assess gingival bleeding and bleeding on probing in patients with leukemia and to verify their correlation with the platelet count. The hypothesis is that patients with leukemia show a strong correlation between these parameters and platelet count.

\section{Methodology Study design and sample}

The target population of this cross-sectional study was patients diagnosed with leukemia, inhabitants of Santa Maria city and the surrounding region, and treated at the Department of HematologyOncology at the University Hospital of Santa Maria (UHSM) in Santa Maria city - one of the three leukemia treatment centers in the state of Rio Grande do Sul in southern Brazil.

Leukemia is a disease with a low incidence in the Brazilian population, ${ }^{6}$ thus sample-size calculation was not considered since it might have required the number of patients to be unobtainable. Therefore, the sample for this study was obtained through a census. The aim was to evaluate all patients in attendance at the hematology-oncology unit at UHSM during the period December 2009 - March 2010. Patients with a definitive diagnosis of any type of leukemia (which, according to World Health Organization, ${ }^{3}$ were: acute myeloid leukemia [AML], chronic myeloid leukemia [CML], acute lymphoid leukemia [ALL] and chronic lymphoid leukemia [CLL]) at any stage of treatment and having a minimum age of 14 years were eligible. Patients who were edentulous, were wearing orthodontic appliances, had psychomotor disturbances, required antibiotic prophylaxis for the examinations, or were using any medication associated with gingival enlargement (nifedipine, cyclosporine, phenytoin) were excluded.

Eligible patients were informed about the purposes of the study, and then invited to participate. Those patients who agreed to participate signed an informed consent form. The study was approved by the Research Ethics Committee, Federal University of Santa Maria, Santa Maria, Brazil.

\section{Measurement reproducibility}

Two examiners (P.D.M.A. and D.A.M.D.) were trained to perform assessments relating to Plaque Index $^{24}$ (PLI), Gingival Index ${ }^{25}$ (GI), and Bleeding on Probing (BOP). They were also calibrated to evaluate Probing Pocket Depth (PPD) and Clinical Attachment Loss (CAL).

Intra- and inter-examiner calibration was done with a millimetric periodontal probe (PC15 Color UNC, Trinity Ind. e Com. Ltda., São Paulo, Brazil). Duplicate examinations at intervals of up to 1 week were performed on 10 patients. Statistical analysis 
of calibration results was accomplished using the kappa test. Measures of reproducibility were also performed during the study.

The initial intraexaminer kappa values were 0.96 (PPD) and 0.86 (CAL) for examiner 1 and 0.93 (PPD) and 0.79 (CAL) for examiner 2. The interexaminer values were 0.92 (PPD) and 0.75 (CAL). During the study, intraexaminer kappa values were 0.97 (PPD) and 0.96 (CAL) for examiner 1 and 0.91 (PPD) and 0.87 (CAL) for examiner 2. The interexaminer values were 0.93 (PPD) and 0.84 (CAL).

\section{Evaluations}

Periodontal parameters (PLI, GI, BOP, PPD and CAL) were assessed at six sites per tooth (mesiobuccal, midbuccal, distobuccal, distolingual, midlingual and mesiolingual), excluding third molars. Examinations were performed in the dental office at UHSM, using a millimetric periodontal probe (PC15 Color UNC, Trinity Ind. e Com. Ltda., São Paulo, Brazil) and a flat mirror.

Clinical attachment loss was defined as the distance from the cementoenamel junction to the bottom of the pocket/sulcus. BOP was evaluated at the base of the pocket together with PPD and measured as present or absent. The measurements (PPD and CAL) were made in millimeters, rounded to the nearest whole millimeter.

Medical data were obtained from the patients' records. Hematological data were collected from the blood tests performed the same day as the periodontal examination. The entire set of hematological tests was processed by the laboratory of clinical analysis of the HUSM. Both investigators were blinded to the counting of blood cells.

\section{Data analysis}

For data descriptive analysis, the means and standard deviations of the respective variables were calculated. Gingival bleeding was based on the average GI score; periodontal bleeding was assessed by the average percentage of sites with BOP. Platelet count was stratified as either less than or equal to $160,000 / \mathrm{mm}^{3}$ or as more than $160,000 / \mathrm{mm}^{3}$. The count of $160,000 / \mathrm{mm}^{3}$ was considered to be within the lower limit for healthy patients. ${ }^{3}$
Pearson's correlation coefficient was used to assess the correlation between GI and stratified platelet count, between BOP and stratified platelet count and between these same parameters for the different types of leukemia. Computer program SPSS v. 13.0 (SPSS Inc., Chicago, USA) was used to analyze the data. Significance level was set at $5 \%$.

\section{Results}

A total of 37 patients who were in treatment for leukemia at UHSM constituted the sample (Figure 1).

Table 1 describes the demographic characteristics and hematological and periodontal parameters of the sample. The mean age was 41.7 years; two thirds were male. Regarding types of leukemia, chronic was more frequent among the myeloid, and acute was more frequent among the lymphoid. The means of PLI and GI were 1.32 and 0.67 , respectively. The means of PPD and CAL were 2.14 and 2.26 , respectively. Thirty percent of sites examined showed BOP present. One third of subjects had platelet counts less than or equal to $160,000 / \mathrm{mm}^{3}$.

Pearson's correlation coefficient between the means of GI and stratified platelet count was $r=-0.003$, which was not statistically significant $(\mathrm{p}=0.987)$. This suggested a null correlation. Pearson's coefficient between the mean percentage of BOP and stratified platelet count showed a weak correlation $(r=0.17)$, but no statistical significance $(\mathrm{p}=0.312)$ (Figure 2). In the different types of leukemia, the correlations between stratified platelet

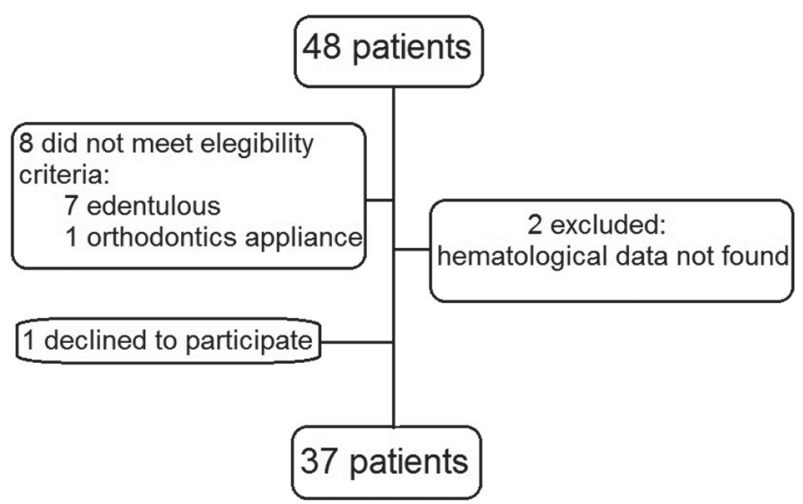

Figure 1 - Flowchart of the study: brief summary of selection of participants until selecting final sample. 
Table 1 - Demographic characteristics and clinical status of patients with leukemia ( $\mathrm{n}=37$ ).

\begin{tabular}{|c|c|}
\hline \multicolumn{2}{|c|}{ Demographic characteristics } \\
\hline Age (years) ${ }^{*}+$ & $41.7( \pm 18.3) 15-80$ \\
\hline \multicolumn{2}{|l|}{ Gender n (\%) } \\
\hline - Female & $11 \quad(29.7)$ \\
\hline - Male & 26 (70.3) \\
\hline \multicolumn{2}{|l|}{ Type of leukemia n (\%) } \\
\hline - AML & $6 \quad(16.2)$ \\
\hline - $\mathrm{CML}$ & 14 (37.8) \\
\hline - ALL & $13 \quad(35.1)$ \\
\hline - $\mathrm{CLL}$ & $4 \quad(10.8)$ \\
\hline \multicolumn{2}{|c|}{ Clinical status } \\
\hline Number of teeth " ${ }^{\dagger}$ & $19.8 \quad( \pm 7.81) 3-28$ \\
\hline Plaque index & $1.32( \pm 0.54)$ \\
\hline Gingival index & $0.67( \pm 0.36)$ \\
\hline $\begin{array}{l}\text { Probing pocket } \\
\text { depth }(\mathrm{mm})^{*}\end{array}$ & $2.14( \pm 0.42)$ \\
\hline Bleeding on probing ${ }^{\pi}$ & $29.91( \pm 16.83)$ \\
\hline $\begin{array}{l}\text { Clinical attachment } \\
\text { loss }(\mathrm{mm})^{*}\end{array}$ & $2.26( \pm 1.71)$ \\
\hline Platelet count $\left(10^{3} / \mathrm{mm}^{3}\right)^{*} \dagger$ & $206.46( \pm 129.4) 37,000-807,000$ \\
\hline - $\leq 160,000$ n (\%) & $12(32.4)$ \\
\hline - > 160,000 n (\%) & $25(67.6)$ \\
\hline
\end{tabular}

${ }^{*}$ Mean $( \pm S D) .{ }^{\pi}$ Mean percentage $( \pm S D) .{ }^{\dagger}$ Minimum and maximum. $\mathrm{AML}$, acute myeloid leukemia; CML, chronic myeloid leukemia; ALL, acute lymphoid leukemia; CLL, chronic lymphoid leukemia.

count and BOP and between platelet count and GI were not statistically significant for either the acute (BOP: $\mathrm{r}=-0.21 ; \mathrm{p}=0.937$; GI: $r=-0.16 ; \mathrm{p}=0.959$ ) or the chronic (BOP: $r=0.307 ; \mathrm{p}=0.188$; GI: $r=0.10 ; \mathrm{p}=0.966$ ) forms of the disease.

\section{Discussion}

The present investigation did not demonstrate significant correlation between either the Gingival Index or the Bleeding on Probing with the platelet count in patients with leukemia.

There is evidence that in leukemia, because of the excessive proliferation of blasts in the bone marrow, there is suppression of the normal hematopoietic cells, causing such problems as anemia, thrombocytopenia, leucopenia, infiltration of organs and tissues and others. Specifically regarding thrombo-

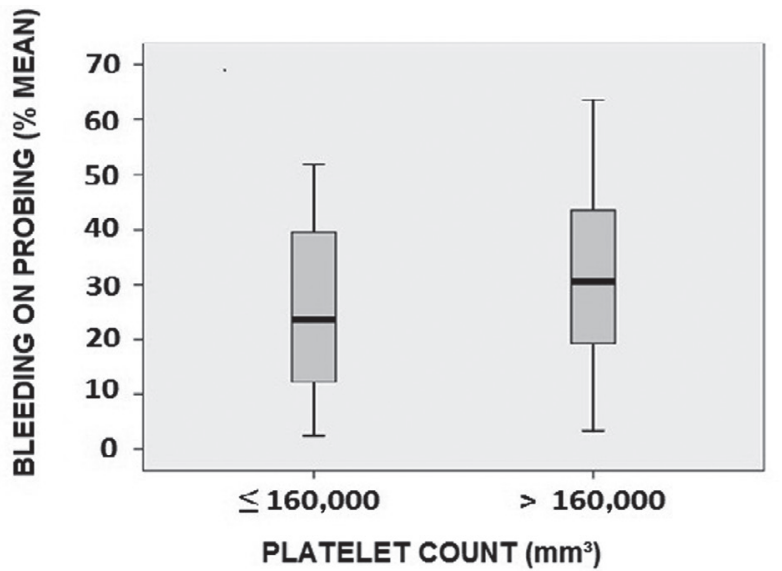

Figure 2 - Box plot demonstrating the relationship between mean percentage of sites with bleeding on probing and platelet count, in two groups: less than or equal to 160,000/ $\mathrm{mm}^{3}$ and more than $160,000 / \mathrm{mm}^{3}$.

cytopenia (low platelet count), an association between increased risk of bleeding and occurrence of hemorrhagic episodes in leukemic patients has been suggested. ${ }^{13-18,20-22}$

While there is no consensus in the literature about the most prevalent oral manifestations in leukemic patients, gingival bleeding, gingival swelling, ulcers and petechiae are the most frequently reported. ${ }^{1,4,5,8-12,16,17,19}$

In patients undergoing active treatment for leukemia, gingival bleeding and petechiae were the most common findings in the oral cavity, occurring twice as often in patients having the acute form of leukemia..$^{13}$ Hou et al. ${ }^{18}$ in a retrospective study of patients at diagnosis, found that gingival bleeding was the second earliest sign of disease. The aforementioned authors ${ }^{13,18}$ identify gingival bleeding as a common finding, especially in the acute forms of leukemia. However, these are retrospective studies in which the data were obtained from medical records often supplied by patients' self-reporting and with no information about periodontal parameters.

In the current study, the mean percentage of sites presenting BOP was approximately $30 \%$. The mean GI was 0.67 , which indicates the presence of localized inflammation in the subgingival regions associated with mild inflammation of the marginal gingiva. The results of mean PLI (1.32) suggest agreement between the accumulation of plaque and the inflam- 
matory parameters (GI, BOP and PPD). The sample consisted of subjects with a mean attachment loss of $2.26 \pm 1.71$ which, according to the American Academy of Periodontology classification, ${ }^{26}$ would be characterized as moderate attachment loss. This finding might be explained by the older age of the sample, since age is considered a risk indicator for attachment loss. ${ }^{27}$

A correlation between periodontal parameters (GI and BOP) and low platelet count was not observed, rejecting our hypothesis. On the other hand, given the knowledge that different forms of leukemia have different progression rates and exhibit distinct prevalence of the oral manifestations, was sought a correlation between the aforementioned parameters and the platelet count in the acute and chronic forms of the disease, separately. Again, no statistically significant associations were found, although it is understood that such analysis due to a small sample have little statistical power.

Meyer et al., ${ }^{20}$ in a cross-sectional study, evaluated three groups of immunocompromised patients, including 53 patients with acute myeloid or lymphoid leukemia. The authors observed a higher prevalence of oral manifestations (mostly ulcers and erythema) in leukemic patients compared with controls. However, in agreement with the results of this study, no association between the periodontal parameters (Gingival Bleeding Index and Plaque Index) and the laboratory data was observed.

In the current study, the lack of correlation between periodontal inflammatory parameters and platelet count might be explained by the limited number of patients which may have been insufficient to express a real correlation. Another aspect to consider is that, in the sample obtained, there was no homogeneous distribution of patients in relation to a platelet count below the normal level (according to the medical literature, less than or equal to $160,000 / \mathrm{mm}^{3}$ ) or to a normal platelet count. Only 12 patients were thrombocytopenic at the time of the exams, with different platelet counts; some of these had levels close to normal, as demonstrated by the mean found. The same finding was reported in the study by Meyer et al., ${ }^{20}$ which may also explain the lack of correlation between periodontal param- eters and laboratory data in this study. In contrast, other studies report either a lower platelet count threshold for oral bleeding or that factors other than platelet count may influence the gingival bleeding episodes. ${ }^{2,15,18,21}$

Moreover, in the present study, patients at any stage of treatment were likely to be sampled, even those who had been discharged from the hospital and that could be stable in their hematological parameters, receiving only medication support and considered controlled. Another aspect to be considered is that our sample consisted of a large number of patients with chronic myeloid leukemia. This type of leukemia, in general, is only third in frequency and, due to its chronic nature, often presents no symptoms and is associated with the absence of oral manifestations. ${ }^{4,5,7,13,14,16,18}$ However, this is understandable in view of the study's eligibility criteria (patients 14 and older), since CML usually occurs in adults.

This study followed a cross-sectional design, evaluating patients with different types of leukemia and in different disease stages. During the study period, the sample that was found, especially with the nonresponse rate of only one patient, is believed to be in agreement with estimated rates of the disease nationally in Brazil (per National Cancer Institute), ${ }^{6}$ which is still considered low. Our data on patients' age and sex are also consistent with the literature showing that leukemia is more common in males at around 40 years of age. ${ }^{67,13,15,22}$ They are also consistent with the prevalence of the different types of disease in the sample.

\section{Conclusions}

From preliminary results it can be concluded that there was no significant correlation between the GI and BOP with the platelet count. Periodontal parameters were in agreement with the degree of plaque accumulation.

\section{Acknowledgments}

This study was supported by CAPES (Coordenação de Aperfeiçoamento de Pessoal de Nível Superior) and FAPERGS (Fundação de Amparo à Pesquisa do Estado do Rio Grande do Sul). 


\section{References}

1. Love AA. Manifestations of leukemia encountered in otolaryngologic and stomatologic practices. Arch Otolaryngol. 1936 Feb; 23(2):173-221.

2. Burke VP, Startzell JM. The leukemias. Oral Maxillofac Surg Clin North Am. 2008 Nov;20(4):597-608.

3. Harris NL, Jaffe ES, Diebold J, Flandrin G, Muller-Hermelink HK, Vardiman JW, et al. The World Health Organization classification of neoplasms of the hematopoietic and lymphoid tissues: report of the Clinical Advisory Committee meeting, Airlie House, VA, November, 1997. Hematol J. 2000;1(1):5366.

4. Ayers KMS, Colquhoun ANK. Leukemia in children. Part I: Orofacial complications and side-effects of treatment. N Z Dent J. 2000 Jun;96(424):60-5.

5. da Silva Santos PS, Fontes A, de Andrade F, de Souza SCOM. Gingival leukemic infiltration as the first manifestation of acute myeloid leukemia. Otolaryngol Head Neck Surg. 2010 Sep;143(3):465-6.

6. Brasil. Ministério da Saúde. Instituto Nacional de Câncer. Estimativa 2010: incidência de câncer no Brasil. [Internet]. Brasília: Ministério da Saúde; 2009 [cited 10 jun. 10]. Avaliable from: http://www.inca.gov.br/estimativa/2010.

7. Lynch MA, Ship II. Initial oral manifestations of leukemia. J Am Dent Assoc. 1967 Oct;75(4):932-40.

8. Wu J, Fantasia JE, Kaplan R. Oral manifestations of acute myelomonocytic leukemia: a case report and review of the classification of leukemias. J Periodontol. 2002;73(6):664-8.

9. Haytac MC, Antmen B, Dogan MC, Sasmaz I. Severe alveolar bone loss and gingival hyperplasia as initial manifestation of Burkitt cell type acute lymphoblastic leukemia. J Periodontol. 2003 Apr;74(4):547-51.

10. Vural F, Ozcan MA, Ozsan GH, Demirkan F, Piskin O, Ates $\mathrm{H}$, et al. Gingival involvement in a patient with CD56+ chronic myelomonocytic leukemia. Leuk Lymphoma. 2004 Feb;45(2):415-8.

11. Ozkan A, Ali R, Ozkalemkas F, Ozcelik T, Ozkocaman V. Acute myeloblastic leukemia (AML-M1) presenting with prominent gingival hypertrophy. Eur J Haematol. 2007 Jun;78(6):547.

12. Au WY, Wong KY, Leung RYY, Tong ACK. Isolated gingival relapse of acute lymphoblastic leukemia after transplantation. J Oral Pathol Med. 2008 Apr;37(4):249-51.

13. Lynch MA, Ship II. Oral manifestations of leukemia: a postdiagnostic study. J Am Dent Assoc. 1967 Nov;75(5):1139-44.

14. Stafford R, Sonis S, Lockhart P, Sonis A. Oral pathoses as diagnostic indicators in leukemia. Oral Surg Oral Med Oral Pathol. 1980 Aug;50(2):134-9.
15. Ashok L, Hema G, Sujatha G. Estimation of salivary amylase and total proteins in leukemia patients and its correlation with clinical feature and radiographic finding. Indian J Dent Res. 2010 Out-Dec;21(4):486-90.

16. Chapple ILC, Saxby MS, Murray JA. Gingival hemorrhage, myelodysplastic syndrome, and acute myeloid leukemia. A case report. J Periodontol. 1999 Oct;70(10):1247-53.

17. Fatahzadeh M, Krakow AM. Manifestation of acute monocytic leukemia in the oral cavity: a case report. Spec Care Dentist. 2008 Sep-Oct;28(5):190-4.

18. Hou GL, Huang JS, Tsai CC. Analysis of oral manifestation of leukemia: a retrospective study. Oral Dis. 1997 Mar;3(1):31-8.

19. Cooper CL, Loewen R, Shore T. Gingival hyperplasia complicating acute myelomonocytic leukemia. J Can Dent Assoc. 2000 Feb;66(2):78-9.

20. Meyer U, Kleinheinz J, Handschel J, Kruse-Lösler B, Weingart D, Joos U. Oral Findings in three different groups of immunocompromised patients. J Oral Pathol Med. 2000 Apr;29(4):153-8.

21. Bergmann OJ, Ellegaard B, Dahl M, Ellegaard J. Gingival status during chemical plaque control with or without prior mechanical plaque removal in patients with acute myeloid leukemia. J Clin Periodontol. 1992 Mar;19(3):169-73.

22. Shankarapillai R, Nair MA, George R, Walsh LJ. Periodontal and gingival parameters in young adults with acute myeloid leukemia in Kerala, South India. Oral Health Prev Dent. 2010;8(4):385-400.

23. Kingman A, Albandar JM. Methodological aspects of epidemiological studies of periodontal disease. Periodontol 2000. 2002;29:11-30.

24. Silness J, Löe H. Periodontal disease in pregnancy II - Correlation between oral hygiene and periodontal conditions. Acta Odontol Scand. 1964 Feb;22:121-35.

25. Löe H. The Gingival Index, the Plaque Index and the Retention Index Systems. J Periodontol. 1967 NovDec;38(6):Suppl:610-6.

26. Armitage GC. Development of a classification system for periodontal diseases and conditions. Ann Periodontol. 1999 Dec;4(1):1-6. Review.

27. Susin C, Dalla Vecchia CF, Oppermann RV, Haugejorden O, Albandar JM. Periodontal attachment loss in an urban population of Brazilian adults: effect of demographic, behavioral, and environmental risk indicators. J Periodontol. 2004 Jul;75(7):1033-41. 